\title{
Antioxidative nanofullerol inhibits macrophage activation and development of osteoarthritis in
} rats

This article was published in the following Dove Press journal: International Journal of Nanomedicine

Yilun Pei ${ }^{1, *}$

Fuai Cuil,2,*

Xuejun Du'

Guowei Shang'

Wanan Xiao'

Xinlin Yang'

Quanjun Cui ${ }^{1}$

'Orthopaedic Research Lab, University of Virginia, Charlottesville, VA, USA;

${ }^{2}$ Department of Biochemistry and Molecular Biology, School of Basic Medical Sciences, Shandong University, Jinan, Shandong, China

*These authors contributed equally to this work
Correspondence: Quanjun Cui

Department of Orthopaedic Surgery,

University of Virginia School of Medicine,

400 Ray C. Hunt Drive, Suite 330,

Charlottesville, VA 22903, USA

Tel + I 4342430266

Fax + I 4342430242

Email qc4q@virginia.edu
Background: There is emerging evidence which suggests that cellular ROS including nitric oxide (NO) are important mediators for inflammation and osteoarthritis (OA). Water-soluble polyhydroxylated fullerene C60 (fullerol) nanoparticle has been demonstrated to have an outstanding ability to scavenge ROS.

Purpose: The objective of this study is to assess the effects of fullerol on inflammation and OA by in vitro and in vivo studies.

Methods: For in vitro experiments, primary mouse peritoneal macrophages and a macrophage cell line RAW264.7 were stimulated to inflammatory phenotypes by lipopolysaccharide (LPS) in the presence of fullerol. For the animal study, OA model was created by intraarticular injection of monoiodoacetate into the knee joints of rats and fullerol was intravenously injected immediately after OA induction.

Results: NO production and pro-inflammatory gene expression induced by LPS was significantly diminished by fullerol in both macrophage cell types. Meanwhile, fullerol could remarkably reduce phosphorylation of p38 mitogen-activated protein kinase, and protein level of transcription factors nuclear factor-kappaB and forkhead box transcription factor 1 within the nucleus. The animal study delineated that systematic administration of fullerol prevented OA, inhibiting inflammation of synovial membranes and the damage toward the cartilage chondrocytes in the OA joints.

Conclusion: Antioxidative fullerol may have a potential therapeutic application for OA.

Keywords: macrophages, inflammation, osteoarthritis, antioxidant, polyhydroxylated fullerene C60

\section{Introduction}

Osteoarthritis (OA) is a debilitating disease characterized by degenerative changes in articular cartilage, bone, and other surrounding tissues. The classical definition of $\mathrm{OA}$ as a wear-and-tear, noninflammatory disease that has recently transitioned to an inflammatory disease based upon a spectrum between normal control and rheumatoid arthritis (RA), an inflammatory joint disease often studied and characterized in comparison with OA. ${ }^{1}$ Recent research has established that the components of both the innate and adaptive immune systems, including multiple cell types, cytokines, chemokines, and complements, play crucial roles in OA pathogenesis. ${ }^{2}$ These components act in concert in the early stage of OA. In this regard, activated macrophages are major cells and key players among these early events and therefore have become a promising target for treatment of $\mathrm{OA}^{2}$ 
Fullerene (C60) and its derivatives have a unique structure, which is composed of 60 carbon atoms, that form a hollow sphere about $1 \mathrm{~nm}$ in diameter. ${ }^{3}$ A variety of their effects have been investigated, such as enzyme inhibition, photo-induced damage toward DNA molecules, cell membranes and cells, microbial and viral inactivation and antioxidation. Among these effects, free radical-scavenging and nitric oxide-quenching properties have shown applications in the biomedical field. Capable of being reversibly reduced by up to 6 electrons and with as many as 34 methyl radicals added to a C60 sphere, fullerenes have been characterized as "radical sponges". ${ }^{4-6}$ The fullerene derivatives that have been investigated for antioxidative properties and cell protective abilities include fullerol (polyhydroxylated fullerene), carboxyfullerene, fullerenecontaining amino acids and peptides. ${ }^{6}$

Fullerol is a water-soluble fullerene derivative which has been demonstrated to function as a powerful antioxidant in different cell types and tissues. ${ }^{6}$ We hypothesized that it could reduce the oxidative level and the inflammatory phenotypes of stimulated macrophages, and therefore it could alleviate the OA symptoms. To test this hypothesis, we herein determined the biological activities of fullerol on mouse primary peritoneal macrophages and RAW264.7 macrophage cell line, as well as its effects on inflammation of synovial membranes and damage of cartilage chondrocytes in an experimental OA model of rat knee joint.

The inflammatory response has been extensively studied by lipopolysaccharide (LPS)-stimulated RAW 264.7 macrophage cells. It has been reported that LPS activates the multiple intracellular signaling pathways involving reactive oxygen species (ROS). ${ }^{7,8}$ Through binding to the toll-like receptor 4 (TLR4) on the surface of macrophages, LPS induces ROS production, leading to stimulation of mitogen-activated protein kinases (MAPKs) and pro-inflammatory transcription factors such as nuclear factor-kappaB (NFkB) and forkhead box transcription factor 1 (FoxO1). ${ }^{7,8}$ The activation of NFkB and FoxO1 subsequently results in elevated expression of the inflammatory markers including tumor necrosis factor-alpha (TNF $\alpha$ ), IL-1 $\beta$, IL-6, induced nitric oxide synthase (iNOS) and nitric oxide (NO) production, that are closely associated with initiation and progression of OA. ${ }^{7-9}$ Furthermore, $\mathrm{p} 38$ MAPK signaling plays an essential role in regulating inflammatory responses. ${ }^{10}$ Therefore, the effect of fullerol on the $\mathrm{p} 38$ MAPK kinase and transcription factors NFkB and FoxO1, as well as TLR4, was assessed in the RAW264 macrophage cell line.

\section{Materials and methods}

\section{Cell culture and treatment}

Primary peritoneal macrophages were isolated from C57/BL6 mouse peritoneum, and cultured in DMEM (Gibco BRL, Gaithersburg, MD, USA) supplemented with $10 \%$ FBS (Hyclone Laboratories, Logan, VT, USA) and $100 \mathrm{IU} / \mathrm{mL}$ penicillin $\mathrm{G}$ and $100 \mu \mathrm{g} / \mathrm{mL}$ streptomycin, in a humidified atmosphere of $5 \%$ carbon dioxide at $37^{\circ} \mathrm{C}$. Mouse RAW264.7 macrophage cell line (ATCC, Manassas, VA, USA) was maintained in the same culture medium. For cell treatment, fullerol (also named as polyhydroxyfullerene or fullerenol with the formulation of $\mathrm{C} 60 \quad(\mathrm{OH})_{24}$; MER Corporation, Tucson, AZ, USA) was added to the culture $0.5 \mathrm{hr}$ prior to addition of $100 \mathrm{ng} / \mathrm{mL}$ LPS (Sigma-Aldrich Co., St Louis, MO, USA) and then cells were co-treated with LPS for $4 \mathrm{hrs}$ for primary macrophages or $24 \mathrm{hrs}$ for RAW264.7 macrophages.

\section{Gene expression analysis}

Total RNA was extracted and purified using an RNeasy kit (QIAGEN Sciences, Valencia, CA, USA) according to the protocol provided by the manufacturer. The RNase-free DNase is used to digest DNA during RNA purification. The purified RNA was stored at $-80^{\circ} \mathrm{C}$. The yield of RNA was determined by measuring absorbance at $260 \mathrm{~nm}$. Synthesis of cDNA from total RNA and the quantitative PCR was carried out by using the iscript ${ }^{\mathrm{TM}} \mathrm{cDNA}$ synthesis kit and the $\mathrm{iQ}^{\mathrm{TM}}$ SYBR Green Supermix kit (Bio-Rad Laboratories, Hercules, CA, USA), respectively. The target genes included $i N O S$, $T N F-\alpha, I L-1 \beta$ and $I L-6$. Gene of 18 s ribosomal RNA was used as an internal control. The threshold cycle (CT) value was calculated from amplification plots. Data were analyzed using the $2^{-\triangle \triangle C T}$ method with $18 \mathrm{~s}$ rRNA serving as the reference. ${ }^{11}$ Gene expression was normalized to the control group in each experiment and represented as fold of change. The primer sequences are listed as follows: 5'-GTGACCAGTTCACTCTTGGT-3' (forward), 5'-CATTGGAAGTGAAGCGTTTCG-3' (reverse) for 18s rRNA, 5'GAGGGATGCCTTCCGCAGCTG-3' (forward), 5'-GAATCGAACCCTGATTCCCCGTC-3' (reverse) for iNOS, 5'CATCTTCTCAAAATTCGAGTGACAA-3' (forward), 5'TGGGAGTAGACAAGGTACAACCC-3' (reverse) for TNF $\alpha$, 5'-CAACCAACAAGTGATATTCTCCATG-3' (forward), 5'-GATCCACACTCTCCAGCTGCA-3' (reverse) for IL-1 $\beta$ and 5'-GAGTCCTTCAGAGAGATACAG-3' (forward), 5'TGGTCTTGGTCCTTAGCC-3' (reverse) for IL-6. 


\section{Western blot analysis}

The cytoplasmic and nuclear proteins were prepared from cells growing on a 6-well plate (approximately 500,000/ well) using the Nuclear and Cytoplasmic Extraction Kit (Thermo Scientific, Waltham, MA, USA). Then, the protein concentration was determined using the Bradford protein assay kit (Bio-Rad Laboratories). The samples containing $100 \mu \mathrm{g}$ proteins were run on $10 \%$ SDS-polyacrylamide gels at a constant current of $80 \mathrm{~V}$ and electro-transferred to nitrocellulose membranes (Thermo Scientific) at a constant voltage of $10 \mathrm{~V}$ overnight. Membranes were blocked with $5 \%$ fatty acid free BSA fraction $\mathrm{V}$ (Roche Diagnostics, Indianapolis, IN, USA) in TBST solution (50 mM Tris, $\mathrm{pH}$ 7.6, $150 \mathrm{mM} \mathrm{NaCl}, 0.05 \%$ Tween 20) for $1 \mathrm{hr}$ at room temperature (RT), washed, and incubated overnight at $4{ }^{\circ} \mathrm{C}$ in 5\% BSA in TBST solution containing each of the specific primary antibodies against TLR4, p-p38, NFkB P65 (Santa Cruz Biotechnology, Dallas, TX, USA), FoxO1 (Cell Signaling), and GAPDH (Sigma-Aldrich Co.). The membranes were then incubated with the corresponding horseradish peroxidase (HRP)-conjugated secondary antibody (Cell Signaling) for $1 \mathrm{hr}$ at RT followed by a chemiluminescent substrate for HRP antibody and enhancer solution (Thermo Scientific) mixed in a 1:1 ratio. The membranes were incubated in the dark with autoradiography films (Genesee Scientific, San Diego, CA, USA), and the films were developed to visualize the bands. Then, the grayscale images (300-400 dpi) on films were scanned using a flat-bed scanner. Densitometry was carried out in Photoshop. Using the magic wand tool from the tool palette, the area of each band was selected, and the mean histogram was recorded and then charted. All Western blots were conducted in triplicates.

\section{Detection of TNF $\alpha$ level}

The amount of TNF $\alpha$ was measured by a commercial ELISA kit (Sigma-Aldrich Co.), following the instructions provided by the manufacturer. Briefly, cells were seeded on a 96-well plate and various treatments were performed. The culture medium was collected and mixed with different assay reagents step by step. The resultant solutions were read at $450 \mathrm{~nm}$ on a microplate reader.

\section{Detection of nitrite production}

The Griess reagent system is based on the chemical reaction which uses sulfanilamide and N-1-napthylethylenediamine dihydrochloride (NED) under acidic (phosphoric acid) conditions. This system detects nitrite production. In the present experiments, nitric oxide detection was performed by a commercial kit (Promega Corporation, Madison, WI, USA), according to the manufacturer's manual. Briefly, all experimental samples and standards containing the dilution series for the nitrite standard reference curve were mixed with $50 \mu \mathrm{L}$ of the sulfanilamide solution and incubated 10 mins at RT without light in a 96-well plate. Then, $50 \mu \mathrm{l}$ of the NED Solution were added to each well and incubated 10 mins at RT without light. The OD was determined at $530 \mathrm{~nm}$ on a microplate reader within 30 mins.

\section{Cytotoxicity assay}

Macrophages in the control group and the treatment groups were growing on a 96-well plate and the cell number was counted by using the WST-1 kit (Fisher Scientific, Waltham, MA, USA). After removal of the supernatant, $150 \mu \mathrm{l}$ new medium was added into each cell. Then, 15 $\mu 1$ Cell Proliferation Reagent WST-1 was added and incubated with cells for $3 \mathrm{hrs}$ in dark with a humidified atmosphere of $5 \%$ carbon dioxide at $37^{\circ} \mathrm{C}$. Each sample was determined at $450 \mathrm{~nm}$ on a microplate reader.

\section{Fluorescent staining of microfilament}

Cells growing on glass coverslips were washed with PBS and fixed with $4 \%$ paraformaldehyde at RT for 10 mins. Fluorescence of microfilament was probed with FITCphalloidin (Sigma-Aldrich Co.) and observed under a Zeiss LSM 880 confocal microscope (Carl Zeiss Meditec AG, Jena, Germany).

\section{Animal experiment}

Female/male 12-14 weeks old Sprague-Dawley rats were used for the animal experiment ( $\mathrm{n}=4 /$ group) with approval of the Institutional Animal Care and Use Committee (IACUC) at University of Virginia, following the Guide for the Care and Use of Laboratory Animals, 8th edition by the U.S. Public Health Service (2010). A well-established OA model of the rat knee joint was induced by intra-articular injection of monoiodoacetate (MIA) $(2 \mathrm{mg} / 50 \mu \mathrm{L}$ PBS). In the treatment group, aqueous nano-fullerol (2 $\mathrm{mg} / \mathrm{kg}$ weight) was administered intravenously immediately after MIA injection. Animals were euthanized at the designated time-points after treatment. The synovial membranes/ joint condyles form both control and injured knees were harvested for histology. 


\section{Statistical analysis}

Data were expressed as mean \pm SD. Statistical evaluation was performed by the ANOVA using the SPSS 15.0 software (SPSS Inc., Chicago, IL, USA). A $P$-value of 0.05 or less was considered as significant difference. A student $t$-test (twotailed and equal variances) was performed to compare the difference between the two groups, and $P<0.05$ was considered significant.

\section{Results}

\section{Mouse primary peritoneal macrophages}

The WST-1 test showed that fullerol had no effect on cell number at the tested doses $(0,0.1,0.3,1,3 \mu \mathrm{M})$ in Figure 1A, indicating no toxicity against the primary macrophages when applied at any dose below $3 \mu \mathrm{M}$. The dose of $1 \mu \mathrm{M}$ was selected for other biological assays, including NO production, mRNA level and production of inflammatory cytokines determined by the Griess reagent, RT-PCR and ELISA, respectively. The LPS group (denoted as LPS) has a higher yield of NO than either the no treatment group (denoted as NT) or the group of LPS combined with fullerol (denoted as LPS + FUL) (Figure 1B). The same pattern has been revealed in the expression of inflammatory-related genes iNOS (Figure 1C), TNF $\alpha$ (Figure 1E), $I L-1 \beta$ and $I L-6$ (Figure $1 \mathrm{~F})$, as well as in the production of TNF $\alpha$ (Figure 1D).

\section{Mouse RAW264.7 macrophage cell line}

RAW 264.7 cell line was derived from peritoneal macrophages and established from an ascites of a tumor induced in a male mouse by intraperitoneal injection of Abselon Leukemia Virus (A-MuLV). It has been widely used for inflammation research because it is very easy to grow and maintain in the lab. As expected, the present study showed that the biological activity of fullerol in the macrophage cell line was similar to that in the primary macrophage (Figure 2). First, fullerol at doses of up to $3 \mu \mathrm{M}$ was not cytotoxic towards RAW267.4 cells determined by the WST-1 assay (Figure 2A). Second, it was found by the Griess reagent test that fullerol could counteract against LPS-stimulated production of NO (Figure 2B). Third, the quantitative RT-PCR analysis revealed that fullerol could significantly suppress LPSinduced increase in the cellular mRNA levels of iNOS, TNF $\alpha$, IL-1 $\beta$, and IL-6 (Figure 2C, E, and F). Last, it was shown by ELISA test that fullerol could inhibit TNF $\alpha$ secretion from cells treated by LPS, with an inhibitory activity comparable to the anti-inflammation drug dexamethasone (Figure 2D). LPS was previously demonstrated to induce RAW264.7 cells to form the multinuclear cells through cell fusion and microfilament re-organization. Furthermore, these multinuclear cells were found to have increased phagocytosis activity and could be viewed by a high-affinity filamentous actin

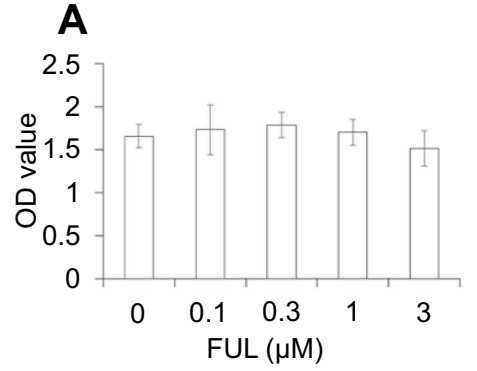

D

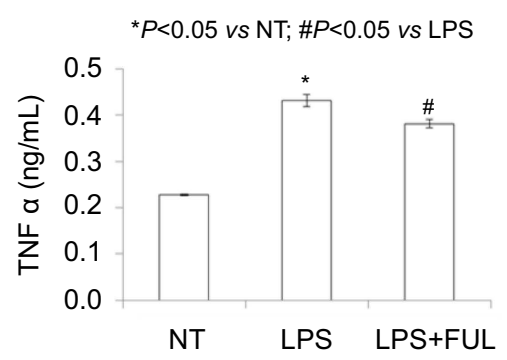

B

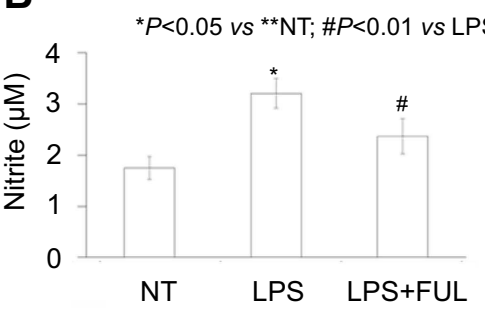

E

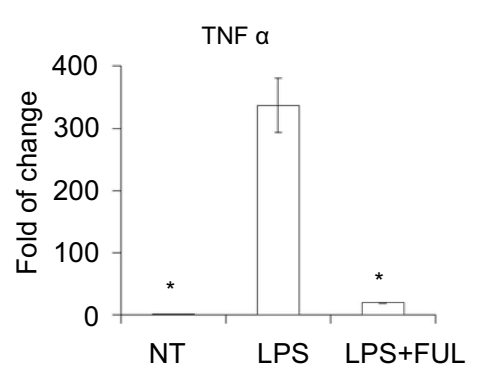

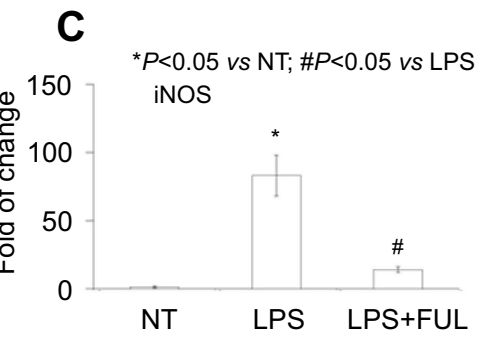

$\mathbf{F}$

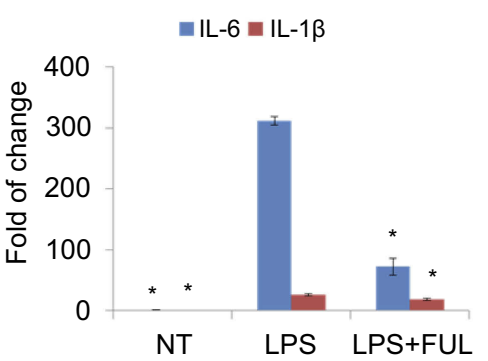

Figure I Fullerol could reduce TNF- $\alpha$, IL-I $\boldsymbol{\beta}$, IL-6 and iNOS expression and nitrite production in primary mouse peritoneal macrophages.

Notes: Fullerol was not toxic at doses of up to $3 \mu \mathrm{M}$ (A, WST-I assay). Fullerol suppressed LPS-induced nitrite production (B, Griess reagent test) and iNOS gene expression (C, quantitative RT-PCR analysis). It also reversed LPS-stimulated production of TNF- $\alpha$ (D, ELISA assay; E, quantitative RT-PCR analysis) and expression of inflammatory cytokines IL-I $\beta$ and IL-6 (F, quantitative RT-PCR analysis). LPS: LPS (100 ng/mL) treatment; LPS + FUL: treatment of LPS (I00 ng/mL) combined with fullerol (I $\mu M)$. Abbreviations: FUL, fullerol; NT, no treatment; iNOS, induced nitric oxide synthase; LPS, lipopolysaccharide; TNF, tumor necrosis factor. 


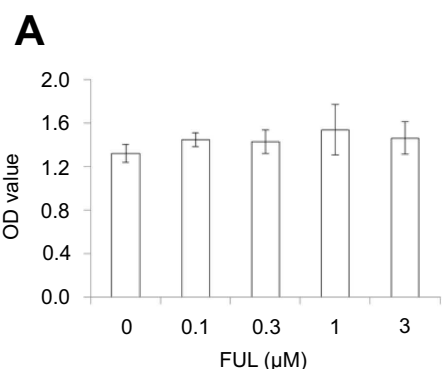

D

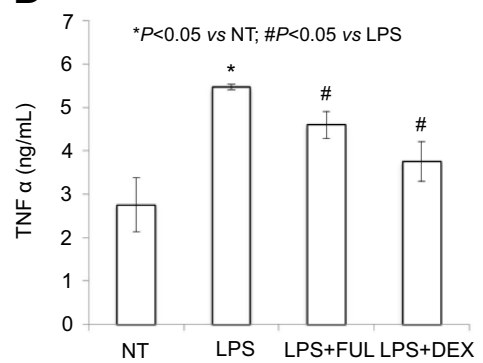

B

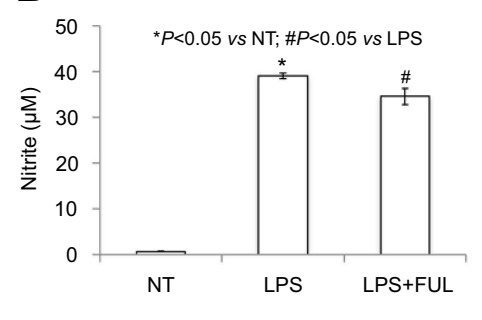

E

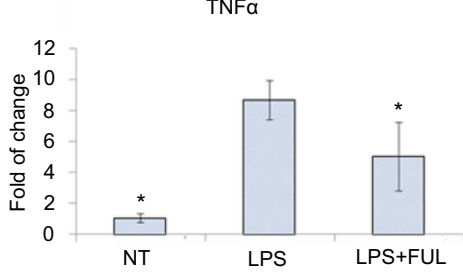

\section{C}

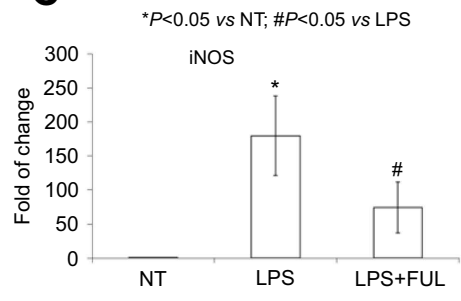

F

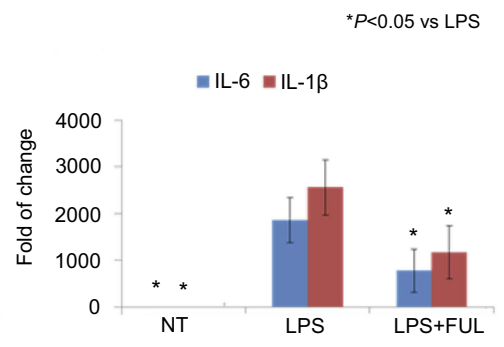

Figure 2 Fullerol could decrease nitrite production and TNF- $\alpha$, IL-6 and IL-I $\boldsymbol{\beta}$ expression in RAW264.7 macrophage cell line.

Notes: Fullerol was not toxic at the doses of up to $3 \mu \mathrm{M}$ (A, WST-I assay). Fullerol suppressed LPS-induced nitrite production (B, Griess reagent test) and iNOS gene expression (C, quantitative RT-PCR analysis). It also reversed LPS-stimulated production of TNF- $\alpha$ (D, ELISA assay; E, quantitative RT-PCR analysis) and expression of inflammatory cytokines IL-I $\beta$ and IL-6 (F, quantitative RT-PCR analysis). LPS: LPS (100 ng/mL) treatment; LPS + FUL: treatment of LPS (I00 ng/mL) combined with fullerol (I $\mu M)$; LPS + DEX: treatment of LPS $(100 \mathrm{ng} / \mathrm{mL})$ combined with dexamethasone $(25 \mu M)$. For all assays, four repeats were performed in each group $(n=4)$.

Abbreviations: FUL, fullerol; NT, no treatment; iNOS, induced nitric oxide synthase; LPS, lipopolysaccharide; TNF, tumor necrosis factor.

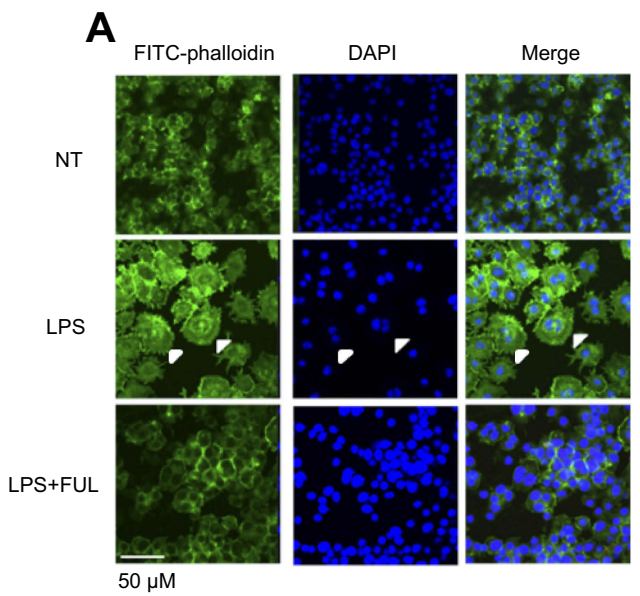

\section{B}

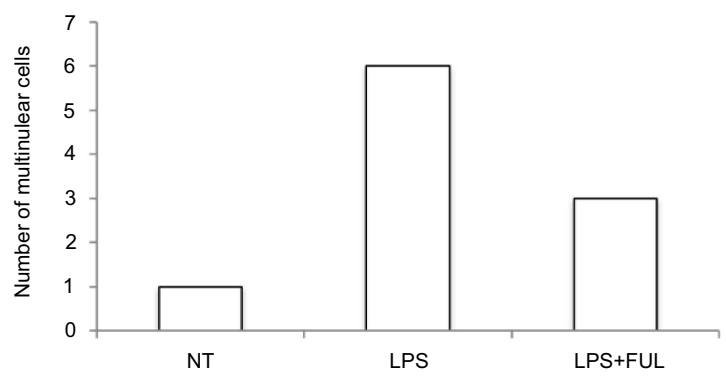

Figure 3 Fullerol suppresses cell fusion and formation of multinuclear cells in RAW264.7 macrophage cell line.

Notes: Cells were stained with the FITC-conjugated phalloidin, a peptide with high ability of binding to the microfilament component F-actin. (A) Images were taken under a fluorescent microscope; (B) numbers of the multinuclear cells in a randomly selected area consisting of 50 nuclei in cell cultures with different treatments. Cell fusion which led to the formation of multinuclear was induced by LPS, and the induction effect of LPS was inhibited with the addition of fullerol. LPS: LPS (I00 ng/mL) treatment; LPS + FUL: treatment of LPS ( $100 \mathrm{ng} / \mathrm{mL})$ combined with fullerol $(\mathrm{I} \mu \mathrm{M})$.

Abbreviations: FUL, fullerol; NT, no treatment; LPS, lipopolysaccharide.

(F-actin) probe phalloidin conjugated to FITC with green fluorescence. ${ }^{12}$ Therefore, we use this technique to provide additional evidence that fullerol could inhibit macrophage activation by LPS. Under a fluorescent microscope, it was obvious that fullerol reversed the cell fusion and microfilament re-organization (white arrows) in LPS-treated cells (Figure $3 \mathrm{~A})$. By counting 50 nuclei in a random area, the number of the multinuclear cells (cells with 3 or more nuclei) was 1, 6 and 3 in NT group, LPS group and LPS + FUL group, respectively (Figure 3B). To explore the mechanisms of fullerol action, the protein level of both cytoplasmic TLR4 and nuclear NFkB was checked by western blot. It was found that addition of fullerol could reduce the production of both molecules stimulated by LPS (Figure 4A and B). Furthermore, two important 

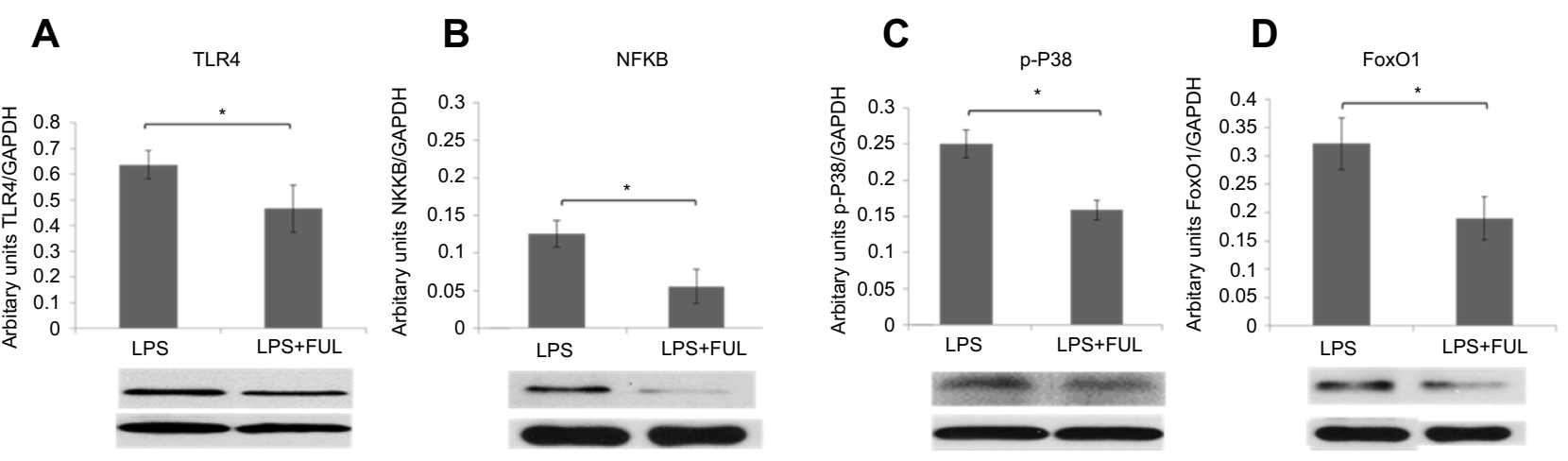

Figure 4 Fullerol decreases protein levels of TLR4 (A) and p-P38 (C) in the cytosol fraction and NFkB (B) and FoxOI (D) in the nuclear fraction determined by western blot. Cells were treated by $100 \mathrm{ng} / \mathrm{mL}$ LPS alone (LPS) or $100 \mathrm{ng} / \mathrm{mL}$ LPS together with I $\mu$ M fullerol (LPS + FUL). It was shown that protein levels of these signal molecules were significantly lower in LPS group than in LPS + FUL group. $* P<0.05, \mathrm{n}=3$.

Abbreviations: FUL, fullerol; FoxOI, forkhead box transcription factor I; LPS, lipopolysaccharide; NFkB, nuclear factor-kappaB; TLR, toll-like receptor.

proteins p38 MAPK and FoxO1 were checked by western blot. The results showed that the group of LPS combined with fullerol had a significantly reduced cytoplasmic level of phosphorylated p38 MAPK (p-p38 MAPK) and nuclear amount of FoxO1, compared to the LPS alone group (Figure 4C and D). Since it has been well established that these proteins are key inflammation-promoting molecules, the current findings indicate that fullerol probably displayed its inhibition on inflammation through the signal pathways involving them.

\section{Animal experiment}

Since the synovium is a main inflammatory site after OA induction, we checked if fullerol can cause histological changes in inflammatory synovium, such as neovascularization stained by H\&E staining. It was reported that fullerol did not show toxicity after intravenous administration of up to $10 \mathrm{mg} / \mathrm{kg}$ body weight to the Sprague-Dawley rats. ${ }^{13}$ In the present pilot animal experiments, fullerol at the dose of $2 \mathrm{mg} /$ $\mathrm{kg}$ weight was administered intravenously immediately after OA induction by MIA injection. As shown in Figure 5, histological analysis demonstrated that treatment of fullerol for 5 days effectively reduced resident fibroblast-like cells and incidence of blood vessels seen in the up/left image of the MIA/ NT group, indicating a relief of the synovial inflammation. It can be seen in Figure 6 that the structures of the joint and articular cartilage were destroyed more seriously at day 21 than at day 11 after MIA injection, and treatment of fullerol for 21 days could counteract loss of matrix, chondrocytes (black arrows) and cartilage thickness (black lines and the bar chart) in the femoral articular cartilage of the injured joint. Particularly, the thicknesses of articular layers randomly selected in PBS group, MIA group and MIA + FUL group were $144 \mu \mathrm{m}, 62 \mu \mathrm{m}$ and $86 \mu \mathrm{m}$, respectively.

\section{Discussion}

In this study, we evaluated the biological activities of fullerol in LPS-stimulated mouse primary peritoneal macrophages and RAW264.7 macrophage cells by measuring production of free radical product nitrite, expression of pro-inflammatory genes and activation of specific intracellular signal molecules. Nitrite test, realtime PCR, ELISA and western blot assays have revealed that LPS can induce macrophage inflammatory phenotypes, and fullerol can remarkably counteract the effects of LPS (Figures 1-4). In addition, we found that fullerol could reduce the synovial inflammation and cartilage degeneration of MIA-induced OA (Figures 5 and 6).

Since the first synthesis of fullerol a quarter century ago, there have been numerous reports about its application in biology and medicine due to its simple preparation and excellent water-soluble property. ${ }^{6,14-26}$ In relevance to the RAW264.7 macrophage cell line, a previous study demonstrated that fullerol at doses of $10-50 \mu \mathrm{M}$ could dose-dependently inhibit ROS production, cell injury and a decrease in mitochondrial membrane potential induced by either sodium nitroprusside or hydrogen peroxide. ${ }^{23}$ Consistently, the current study unveiled that even at lower doses ( $1 \mu \mathrm{M}$ or less) fullerol was able to prevent NO production after LPS treatment. Accordingly, fullerol suppressed LPS-stimulated expression of inflammation-related genes $i N O S, T N F-\alpha, I L-1 \beta$ and IL- 6 determined by RT-PCR and ELISA assays 


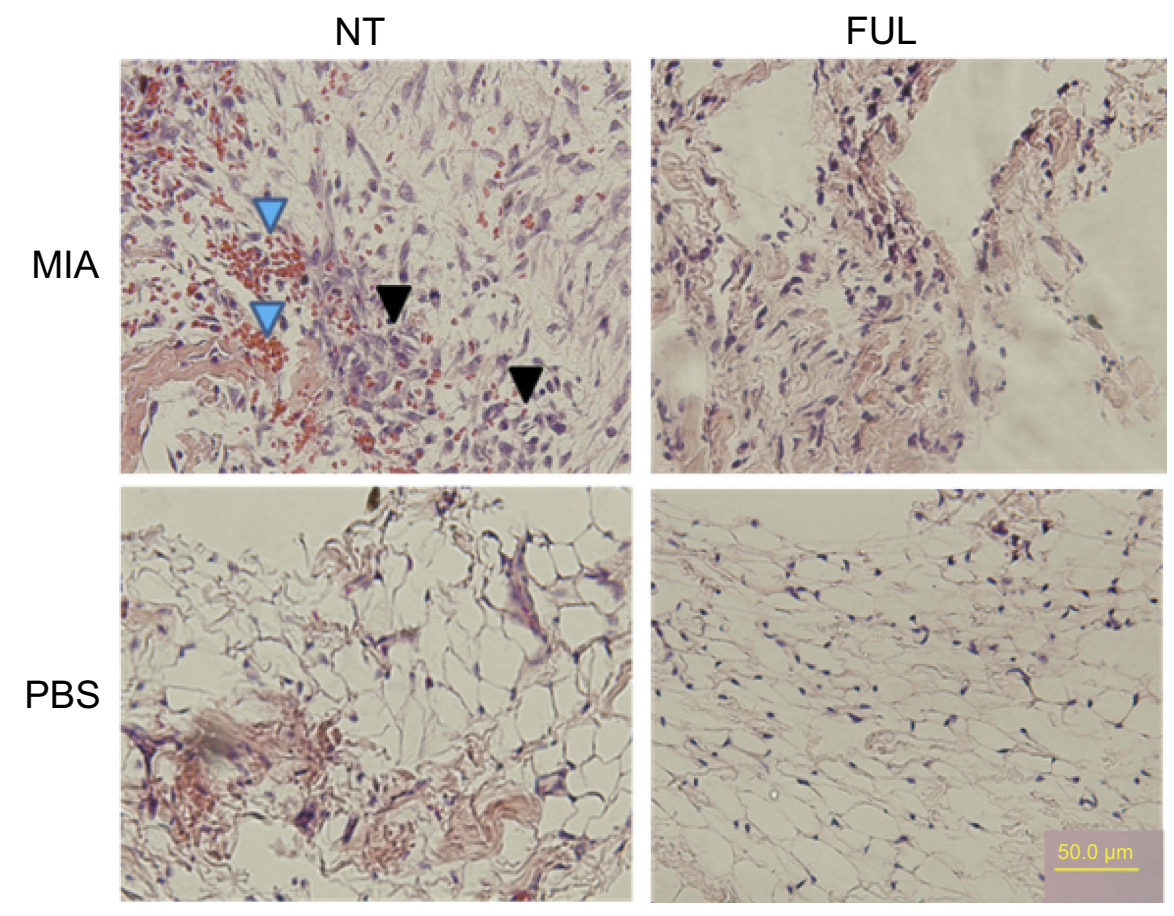

Figure 5 Representative H\&E staining of synovium from rat knee joints (control: PBS, injured: MIA) with (FUL) or without (NT) fullerol treatment for 5 days.

Notes: Fullerol of $2 \mathrm{mg} / \mathrm{kg}$ weight was administered intravenously immediately after MIA injection. The results showed that fullerol reduces red blood cells (blue arrows) and fibroblast-like cells (black arrows) in the synovium of the injured knee seen in the up/left image of the MIA/NT group.

Abbreviations: FUL, fullerol; MIA, monoiodoacetate; NT, no treatment.

A
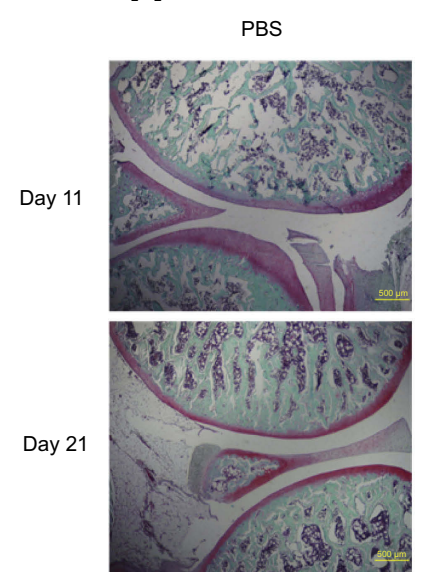
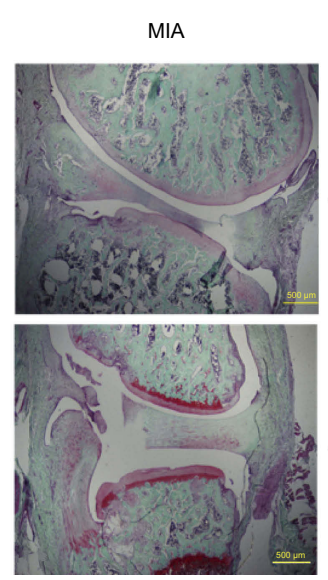

B

PBS/NT
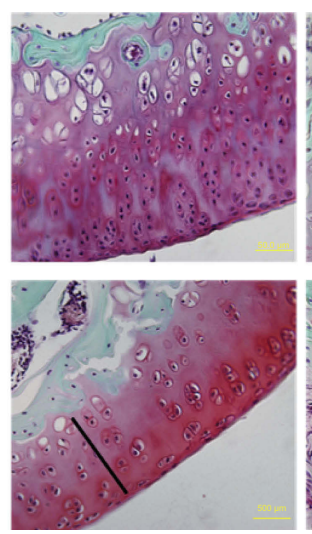

MIA/NT
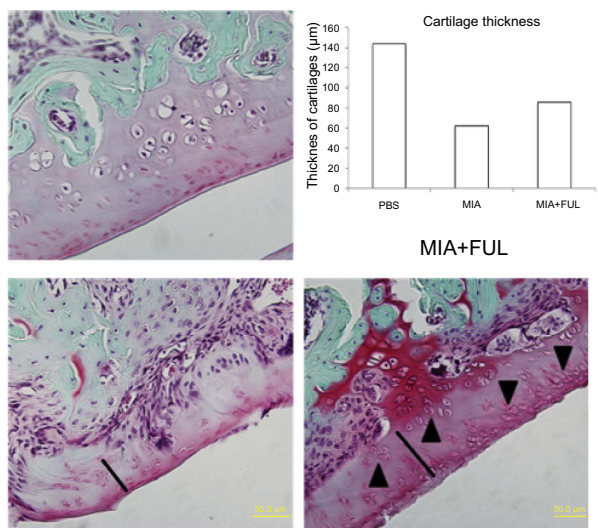

Figure 6 Representative Safranin O/Fast green staining of rat knee joints (A, control: PBS, injured: MIA) and femoral articular cartilages (B) in both joints with (Ful) or without (NT) fullerol treatment.

Notes: Osteoarthritis was induced in the knee joints by intra-articular injection of MIA for up to 21 days. It was shown that MIA-induced histopathological alterations, including a decrease in the matrix production and the thickness of the articular cartilage, erosion of the articular cartilage, disorientation of the chondrocytes, fibrillation of the superficial zone and exposure of the subchondral bone, were time-dependent (A). Fullerol alleviates the loss of matrix, chondrocytes (black arrows) and cartilage thickness (black lines and the bar chart) in the femoral articular cartilage (B).

Abbreviations: MIA, monoiodoacetate; NT, no treatment.

(Figure 2), as well as the formation of multi-nucleated macrophages by fluorescent staining of cellular microfilaments using FITC-labeled phalloidin (Figure 3). More importantly, in primary peritoneal macrophages, the anti-inflammatory activity was further confirmed by the experimental data about inhibition of fullerol on the production of both NO and pro-inflammatory cytokines in the presence of LPS (Figure 1).

It has been established that intracellular ROS is pivotal mediators of signal transduction after LPS stimulation in 
RAW264.7 cells. ${ }^{7,8}$ Binding of LPS to its specific receptor TLR4 triggers a series of biochemical cascades within cells, including: 1) overloaded cellular ROS, 2) activation of various MAPKs, 3) phosphorylation of different transcription factors, and 4) regulation of target gene expression (Figure 7). ${ }^{7,8}$ In the present study, for the first time we found that fullerol effectively inhibited phosphorylation of p38 MAPK, nuclear localization of transcription factors NFKB and FoxO1 by western blot and TLR4 mRNA level by RT-PCR (Figures 3 and 4). Based on these data, we proposed a putative mechanism underlying the antiinflammatory effects of fullerol (Figure 7). Interestingly, a decrease in iNOS mRNA level implied that fullerene derivatives probably suppressed NO production not only through regulation of the enzymatic activity, ${ }^{27}$ but also the biosynthesis of the iNOS which is a key enzyme to NO production. In addition, the result related to regulation of FoxO1 and TLR4 is supported by a recent report, which suggested that TLR4 was a target gene of FoxO1 in

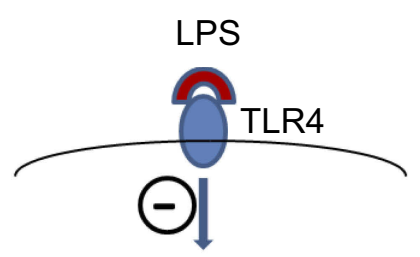

ROS/RNS

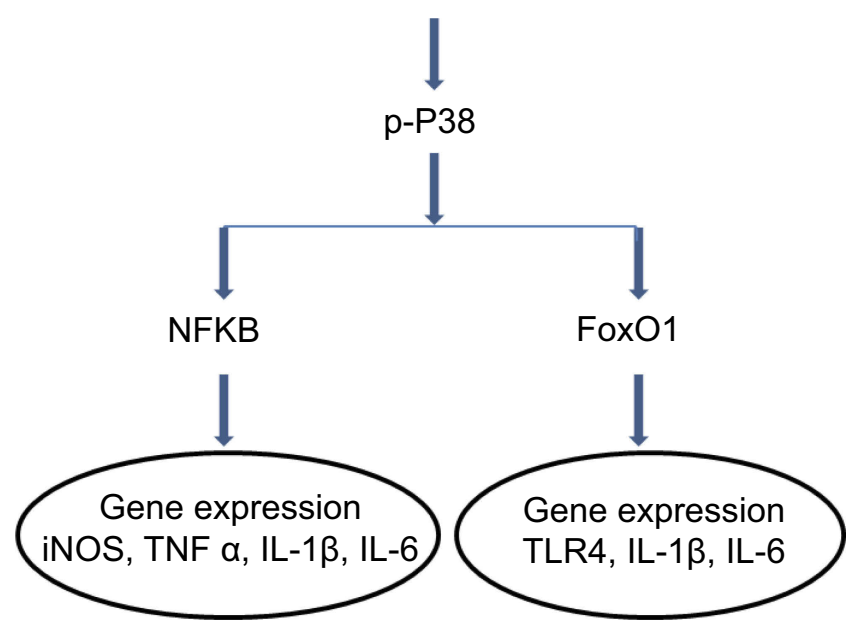

Figure 7 A proposed mechanism underlying inhibitory effects of fullerol on inflammation-related gene expression in LPS-activated macrophages. The symbol $\square$ represents inhibition of fullerol against free radicals produced after binding of LPS to its ligand TLR4. The p38 MAPK plays a pivotal role in the regulation. Through transcription factors, NFkB and FoxOI, the expression of targeted genes was regulated. The decrease in iNOS and TLR4 expression reduces the cellular level of reactive nitrogen species (RNS) and TLR4 protein, which further minimizes the inflammatory effects of LPS. It is worth noting that other signal pathways might also be involved in the anti-inflammation of fullerol.

Abbreviations: FoxOI, forkhead box transcription factor I; iNOS, induced nitricoxide synthase; LPS, lipopolysaccharide; NFkB, nuclear factor-kappaB; TLR, toll-likereceptor.
RAW264.7 cells stimulated by LPS. ${ }^{9}$ Furthermore, the proposed antioxidant mechanism in the anti-inflammatory effect of fullerol is consistent with the previous findings on other oxidants such as $\mathrm{N}$-acetyl cysteine, resveratrol and malvidin. ${ }^{8,28,29}$ However, the complexity of the involved signaling networks made it hard to distinguish between cause and consequence and to identify upstream and downstream events. In this regard, the detailed mechanism is currently far from being fully clarified and apparently a lot of research is required in the future.

The MIA model is a fast-progressing biochemically induced model of OA. MIA induces chondrocyte death in rodent and non-rodent species and causes loss of articular cartilage with subchondral bone lesions that mimic human OA. The MIA model is simple to induce, with good reproducibility, therefore is frequently used for the "proof of concept" study of OA. ${ }^{30-35}$ In particular, macrophage activation and increased levels of $\mathrm{NO}$ and proinflammatory cytokines such as IL- $1 \beta$ and IL-6 within the knee joints have been described using this model. $^{30,34,35}$ The encouraging outcomes from our in vitro experiments suggest that fullerol is an effective inflammation inhibitor (Figures 1 and 2) and enabled us to conduct a pilot study to assess the efficacy of fullerol on protecting the joints from $\mathrm{OA}$ inflammation and injuries with the MIA model. Indeed, the present histological analysis indicated that a single injection of fullerol, systematically administered to rats, effectively decreased the synovial inflammation and the cartilage degeneration in the rat OA knee joint (Figures 5 and 6). The result is in accordance with the reports of Yudoh et al, describing the inhibitory effects of a water-soluble fullerene (polyvinylpyrrolidone-entrapped C60) on the surgically induced OA of the rabbit knee joints and adjuvant-induced RA of the rat knee joints. ${ }^{36,37}$ It is worth noting that the fullerol (polyhydroxylated C60) used in our experiments is different from the pristine $\mathrm{C} 60$, and preparation of fullerol is much easier than the pristine C60 for biomedical application. In addition, the animal model in our study and theirs are different, therefore, a comparative study between the two drugs will be more meaningful using the same animal model.

Various cellular ROS including NO are particularly damaging to the articular cartilage matrix components through direct attack, reduced synthesis, or activation of matrix-metalloproteinases. ${ }^{38,39}$ Furthermore, ROS may contribute to cartilage degradation, joint inflammation, chondrocyte apoptosis, and alteration of redox-sensitive 
intracellular pathways. ${ }^{39}$ Injurious compression of articular cartilage was found sufficient to produce chondrocyte apoptosis, which was partially mediated by ROS production. ${ }^{40}$ ROS cause damage to mitochondrial membranes, which leads to release of caspases and other pro-apoptotic molecules. ${ }^{41}$ The finding that fullerol is able to decrease ROS produced by active macrophages (Figures 1 and 2), along with our published data on the protection of fullerol against cell death and matrix degeneration of chondrocytes induced by hydrogen peroxide and IL- $1,{ }^{20}$ suggests that fullerol is more likely to improve the OA symptom through its activities on both macrophages and chondrocytes in the joint tissues. This is further supported by the experiments of Yudoh et al, revealing that the tested water-soluble fullerene could prevent stress-induced downregulation of matrix production, and apoptosis and premature senescence in human chondrocytes. $^{37}$

There are several limitations in the current study. First, although the rodent peritoneal macrophage has been frequently applied for the research on joint inflammatory diseases, it is not the exact cell type which resides in the joint tissues. ${ }^{42,43}$ Considering the rodent joint macrophages are difficult to obtain, inclusion of the macrophages from the synovial tissues of human OA patients may be a good choice. ${ }^{36,42}$ Secondly, as mentioned above, the MIA model is good for the "proof of concept" study. However, its limitation is obvious since the OA progression of this model is much faster than the surgically injured model which is closer to mimic the OA disease in human. Therefore, evaluation of fullerol is under investigation in our lab, using the OA model induced by the anterior cruciate ligament transection. ${ }^{38,44}$ In addition, in the present experiments fullerol was administered simultaneously with the induction of OA, and only the preventive activity of fullerol was studied. In this sense, the current findings are very preliminary and additional experiments should be undertaken in which fullerol treatment will be performed at different time-points of OA according to the similar research previously published. ${ }^{45}$ Furthermore, local injection is considered as the way preferentially chosen for pharmaceutical treatment of the knee OA in the clinic. $^{46,47}$ Intraarticular administration should be a better choice than the intravenous injection to assess the efficacy of fullerol in the therapy of OA. Lastly, more detailed investigation and analysis of the pathway and animal experiments are further required to strengthen the conclusion obtained from the current study.

\section{Conclusion}

The current study reveals for the first time that fullerol can inhibit the inflammatory response by reducing ROS production and down-regulating expression of inflammatory genes possibly via ROS/p38 MAPK/NFkB and ROS/p38 MAPK/FoxO1 pathways. Fullerol can inhibit progression of experimental OA in rats and thus might be potentially used as a new anti-inflammatory drug for treatment of OA.

\section{Acknowledgments}

The work supported by the NIH/National Institute of Arthritis and Musculoskeletal and Skin Diseases grant No. 5R21AR070987 and by Research fund from University of Virginia School of Medicine.

\section{Disclosure}

QC reports grants from the NIH the University of Virginia, and Exactech. The authors report no other conflicts of interest in this work.

\section{References}

1. de Lange-Brokaar BJ, Ioan-Facsinay A, van Osch GJ, et al. Synovial inflammation, immune cells and their cytokines in osteoarthritis: a review. Osteoarthritis Cartilage/OARS Osteoarthritis Res Soc. 2012;20(12):1484-1499. doi:10.1016/j.joca.2012.08.027

2. Kandahari AMYX, Dighe AS, Pan D, Cui Q. Recognition of immune response for the early diagnosis and treatment of osteoarthritis. J Immunol Res. 2015;2015(2015):1-13. doi:10.1155/2015/192415

3. Kroto HW, Heath JR, Obrien SC, Curl RF, Smalley RE. C-60 - buckminsterfullerene. Nature. 1985;318(6042):162-163. doi:10.1038/ 318162a0

4. Krusic PJ, Wasserman E, Keizer PN, Morton JR, Preston KF. Radical reactions of C60. Science. 1991;254(5035):1183-1185. doi:10.1126/ science.254.5035.1183

5. Mcewen CN, Mckay RG, Larsen BS. C-60 as a radical sponge. $J$ Am Chem Soc. 1992;114(11):4412-4414. doi:10.1021/ja00037a064

6. Yang X, Ebrahimi A, Li J, Cui Q. Fullerene-biomolecule conjugates and their biomedicinal applications. Int J Nanomedicine. 2014;9:77-92. doi:10.2147/IJN.S52829

7. Emre Y, Hurtaud C, Karaca M, Nubel T, Zavala F, Ricquier D. Role of uncoupling protein UCP2 in cell-mediated immunity: how macrophage-mediated insulitis is accelerated in a model of autoimmune diabetes. Proc Natl Acad Sci USA. 2007;104(48):19085-19090. doi:10.1073/pnas.0709557104

8. Bognar E, Sarszegi Z, Szabo A, et al. Antioxidant and anti-inflammatory effects in RAW264.7 macrophages of malvidin, a major red wine polyphenol. PLoS One. 2013;8(6):e65355. doi:10.1371/journal.pone.0065355

9. Gupta P, Srivastav S, Saha S, Das PK, Ukil A. Leishmania donovani inhibits macrophage apoptosis and pro-inflammatory response through AKT-mediated regulation of beta-catenin and FOXO-1. Cell Death Differ. 2016;23(11):1815-1826. doi:10.1038/cdd.2016.101

10. Yang Y, Kim SC, Yu T, et al. Functional roles of $\mathrm{p} 38$ mitogen-activated protein kinase in macrophage-mediated inflammatory responses. Mediators Inflamm. 2014;2014:352371. doi:10.1155/ $2014 / 352371$ 
11. Livak KJ, Schmittgen TD. Analysis of relative gene expression data using real-time quantitative PCR and the $2^{-\triangle \Delta C T}$ method. Methods. 2001;25(4):402-408. doi:10.1006/meth.2001.1262

12. Nakanishi-Matsui M, Yano S, Matsumoto N, Futai M. Lipopolysaccharide induces multinuclear cell from RAW264.7 line with increased phagocytosis activity. Biochem Biophys Res Commun. 2012;425(2):144-149. doi:10.1016/j.bbrc.2012.07.050

13. Monteiro-Riviere NA, Linder KE, Inman AO, Saathoff JG, Xia XR, Riviere JE. Lack of hydroxylated fullerene toxicity after intravenous administration to female Sprague-Dawley rats. $J$ Toxicol Environ Health Part A. 2012;75(7):367-373. doi:10.1080/15287394.2012.67 0894

14. Li J, Takeuchi A, Ozawa M, Li XH, Saigo K, Kitazawa K. C-60 fullerol formation catalyzed by quaternary ammonium hydroxides. $J$ Chem Soc Chem Comm. 1993;(23):1784-1785.

15. Chiang LY, Upasani RB, Swirczewski JW, Soled S. Evidence of hemiketals incorporated in the structure of fullerols derived from aqueous acid chemistry. $J$ Am Chem Soc. 1993;115(13):5453-5457. doi:10.1021/ja00066a014

16. Kovac T, Borisev I, Crevar B, et al. Fullerol $\mathrm{C} 60(\mathrm{OH}) 24$ nanoparticles modulate aflatoxin B1 biosynthesis in Aspergillus flavus. Sci Rep. 2018;8 (1):12855. doi:10.1038/s41598-018-31305-9

17. Jin L, Ding M, Oklopcic A, et al. Nanoparticle fullerol alleviates radiculopathy via NLRP3 inflammasome and neuropeptides. Nanomed. 2017;13 (6):2049-2059. doi:10.1016/j.nano.2017.03.015

18. Arifa RDN, Paula TP, Madeira MFM, et al. The reduction of oxidative stress by nanocomposite Fullerol decreases mucositis severity and reverts leukopenia induced by Irinotecan. Pharmacol Res. 2016;107:102-110. doi:10.1016/j.phrs.2016.03.004

19. Yang X, Li CJ, Wan Y, Smith P, Shang G, Cui Q. Antioxidative fullerol promotes osteogenesis of human adipose-derived stem cells. Int J Nanomedicine. 2014;9:4023-4031. doi:10.2147/IJN.S66785

20. Yang X, Jin L, Yao L, Shen FH, Shimer AL, Li X. Antioxidative nanofullerol prevents intervertebral disk degeneration. Int J Nanomedicine. 2014;9:2419-2430. doi:10.2147/IJN.S60853

21. Liu HJ, Yang XL, Zhang Y, Dighe A, Li XD, Cui QJ. Fullerol antagonizes dexamethasone-induced oxidative stress and adipogenesis while enhancing osteogenesis in a cloned bone marrow mesenchymal stem cell. J Orthop Res. 2012;30(7):1051-1057. doi:10.1002/ jor.22054

22. Tang J, Chen Z, Sun B, et al. Polyhydroxylated fullerenols regulate macrophage for cancer adoptive immunotherapy and greatly inhibit the tumor metastasis. Nanomed. 2016;12(4):945-954. doi:10.1016/j. nano.2015.11.021

23. Chen YW, Hwang KC, Yen CC, Lai YL. Fullerene derivatives protect against oxidative stress in RAW 264.7 cells and ischemia-reperfused lungs. Am J Physiol Regul Integr Comp Physiol. 2004;287(1):R21R26. doi:10.1152/ajpregu.00310.2003

24. Roy P, Bag S, Chakraborty D, Dasgupta S. Exploring the inhibitory and antioxidant effects of fullerene and fullerenol on ribonuclease A. ACS Omega. 2018;3(9):12270-12283. doi:10.1021/acsomega.8b01584

25. Hao T, Li J, Yao F, et al. Correction to injectable fullerenol/alginate hydrogel for suppression of oxidative stress damage in brown adipose-derived stem cells and cardiac repair. ACS Nano. 2018;12 (10):10564. doi:10.1021/acsnano.8b02946

26. Li J, Lei R, Li X, et al. The antihyperlipidemic effects of fullerenol nanoparticles via adjusting the gut microbiota in vivo. Part Fibre Toxicol. 2018;15(1):5. doi:10.1186/s12989-018-0241-9

27. Satoh M, Takayanag I. Pharmacological studies on fullerene (C-60), a novel carbon allotrope, and its derivatives. J Pharmacol Sci. 2006;100(5):513-518.

28. David J, Nandakumar A, Muniroh M, Akiba S, Yamamoto M, Koriyama C. Suppression of methylmercury-induced MIP-2 expression by N-acetyl-L-cysteine in murine RAW264.7 macrophage cell line. Eur J Med Res. 2017;22(1):45. doi:10.1186/ s40001-017-0287-4
29. Murakami Y, Kawata A, Ito S, Katayama T, Fujisawa S. The radical scavenging activity and cytotoxicity of resveratrol, orcinol and 4-allylphenol and their inhibitory effects on cox-2 gene expression and Nf-kappab activation in RAW264.7 cells stimulated with porphyromonas gingivalis-fimbriae. In Vivo. 2015;29(3):341-349.

30. Yang X, Chordia MD, Du X, et al. Targeting formyl peptide receptor 1 of activated macrophages to monitor inflammation of experimental osteoarthritis in rat. $J$ Orthopaedic Res. 2016;34(9):1529-1538. doi: $10.1002 /$ jor. 23148

31. Cinar BM, Ozkoc G, Bolat F, Karaeminogullari O, Sezgin N, Tandogan RN. Intra-articular zoledronic acid in a rat osteoarthritis model: significant reduced synovitis may indicate chondroprotective effect. Knee Surg Sports Traumatol Arthroscopy. 2015;23 (5):1410-1418. doi:10.1007/s00167-014-2955-z

32. Nam J, Perera P, Liu J, et al. Sequential alterations in catabolic and anabolic gene expression parallel pathological changes during progression of monoiodoacetate-induced arthritis. PLoS One. 2011;6(9): e24320. doi:10.1371/journal.pone.0024320

33. Udo M, Muneta T, Tsuji K, et al. Monoiodoacetic acid induces arthritis and synovitis in rats in a dose- and time-dependent manner: proposed model-specific scoring systems. Osteoarthritis Cartilage/ OARS Osteoarthritis Res Soc. 2016;24(7):1284-1291. doi:10.1016/j. joca.2016.02.005

34. Lee J, Hong YS, Jeong JH, et al. Coenzyme Q10 ameliorates pain and cartilage degradation in a rat model of osteoarthritis by regulating nitric oxide and inflammatory cytokines. PLoS One. 2013;8(7): e69362. doi:10.1371/journal.pone.0069362

35. Wang ZM, Chen YC, Wang DP. Resveratrol, a natural antioxidant, protects monosodium iodoacetate-induced osteoarthritic pain in rats. Biomed Pharmacother. 2016;83:763-770. doi:10.1016/j.biopha.2016.06.050

36. Yudoh K, Karasawa R, Masuko K, Kato T. Water-soluble fullerene (C60) inhibits the osteoclast differentiation and bone destruction in arthritis. Int $J$ Nanomedicine. 2009;4:233-239.

37. Yudoh K, Shishido K, Murayama H, et al. Water-soluble c60 fullerene prevents degeneration of articular cartilage in Osteoarthritis via down-regulation of chondrocyte catabolic activity and inhibition of cartilage degeneration during disease development. Arthritis Rheum. 2007;56(10):3307-3318. doi:10.1002/art.22917

38. Castro RR, Cunha FQ, Silva FS Jr., Rocha FA. A quantitative approach to measure joint pain in experimental osteoarthritis-evidence of a role for nitric oxide. Osteoarthritis Cartilage/OARS Osteoarthritis Res Soc. 2006;14(8):769-776. doi:10.1016/j.joca.2006.01.013

39. Henrotin YE, Bruckner P, Pujol JP. The role of reactive oxygen species in homeostasis and degradation of cartilage. Osteoarthritis Cartilage/OARS Osteoarthritis Res Soc. 2003;11(10):747-755. doi:10.1016/S1063-4584(03)00150-X

40. Kurz B, Lemke A, Kehn M, et al. Influence of tissue maturation and antioxidants on the apoptotic response of articular cartilage after injurious compression. Arthritis Rheum. 2004;50(1):123-130. doi: $10.1002 /$ art. 11438

41. Orrenius S. Reactive oxygen species in mitochondria-mediated cell death. Drug Metab Rev. 2007;39(2-3):443-455. doi:10.1080/ 03602530701468516

42. Papathanassiu AE, Ko JH, Imprialou M, et al. BCAT1 controls metabolic reprogramming in activated human macrophages and is associated with inflammatory diseases. Nat Commun. 2017;8:16040. doi: $10.1038 /$ ncomms 16040

43. Sugita R, Kuwabara H, Sugimoto K, et al. A novel selective prostaglandin E2 synthesis inhibitor relieves pyrexia and chronic inflammation in rats. Inflammation. 2016;39(2):907-915. doi:10.1007/ s10753-016-0323-5

44. Akasaki Y, Matsuda S, Nakayama K, Fukagawa S, Miura H, Iwamoto Y. Mevastatin reduces cartilage degradation in rabbit experimental osteoarthritis through inhibition of synovial inflammation. Osteoarthritis Cartilage/OARS Osteoarthritis Res Soc. 2009;17 (2):235-243. doi:10.1016/j.joca.2008.06.012 
45. Mohan G, Perilli E, Parkinson IH, Humphries JM, Fazzalari NL, Kuliwaba JS. Pre-emptive, early, and delayed alendronate treatment in a rat model of knee osteoarthritis: effect on subchondral trabecular bone microarchitecture and cartilage degradation of the tibia, bone/ cartilage turnover, and joint discomfort. Osteoarthritis Cartilage/ OARS Osteoarthritis Res Soc. 2013;21(10):1595-1604. doi:10.1016/ j.joca.2013.06.020
46. Huskisson EC, Donnelly S. Hyaluronic acid in the treatment of osteoarthritis of the knee. Rheumatology (Oxford). 1999;38(7):602-607.

47. Ayhan E, Kesmezacar H, Akgun I. Intraarticular injections (corticosteroid, hyaluronic acid, platelet rich plasma) for the knee osteoarthritis. World J Orthop. 2014;5(3):351-361. doi:10.5312/wjo.v5.i3.351

\section{Publish your work in this journal}

The International Journal of Nanomedicine is an international, peerreviewed journal focusing on the application of nanotechnology in diagnostics, therapeutics, and drug delivery systems throughout the biomedical field. This journal is indexed on PubMed Central, MedLine, CAS, SciSearch ${ }^{\mathbb{R}}$, Current Contents ${ }^{\mathbb{B}} /$ Clinical Medicine,
Journal Citation Reports/Science Edition, EMBase, Scopus and the Elsevier Bibliographic databases. The manuscript management system is completely online and includes a very quick and fair peer-review system, which is all easy to use. Visit http://www.dovepress.com/ testimonials.php to read real quotes from published authors.

Submit your manuscript here: https://www.dovepress.com/international-journal-of-nanomedicine-journal 